\title{
Preface to the special issue on optimization with polynomials and tensors
}

\author{
Jiawang $\mathrm{Nie}^{1}$ \\ Published online: 12 March 2020 \\ (c) Springer Science+Business Media, LLC, part of Springer Nature 2020
}

This special issue collects latest research results about the topics of polynomial optimization and tensor computation. This is a vibrant new research area. It has gathered broad, strong interests from both pure and applied mathematicians, scientists and engineers. When compared to traditional optimization topics, polynomial and tensor optimization is highly challenging in regard to global optimality, nonnegativity or positivity certificates for polynomials, tensor complementarity, tensor decompositions with various structures, and certificates for special tensors. These computational challenges are desperately in need of powerful tools from algebra, convex geometry, numerical linear algebra, and semidefinite optimization. A common research difficulty in polynomial and tensor optimization is the lack of efficient certificates for nonnegative polynomials. However, sum of squares relaxations and semidefinite programming provide fast, practical and reliable methods for nonnegativity certificates. As a result, many hard optimization problems about polynomials and tensors can be solved successfully by the Moment-SOS hierarchy of semidefinite programming relaxations. To get efficient methods for solving polynomial and tensor optimization, we also need to master intrinsic properties of polynomials and tensors. Tensor computation gives big impetus to polynomial optimization, while polynomial optimization provides efficient and reliable methods for tensor computation. This special issue has a good collection of latest results about these aspects of polynomial and tensor optimization.

The articles selected in this special issue cover broad topics of polynomial and tensor optimization. They are about convexity in polynomial optimization, entanglements in tensors, neural networks for tensor decompositions, polynomial systems, tensor eigenvalues and low rank approximations, saddle point problems for rational polynomials, sum of square relaxations, stochastic tensor complementarity, detection of $\mathrm{P}\left(\mathrm{P}_{0}\right)$ tensors, orthogonal tensor decompositions, tensor spectral $p$-norm and tensor partitions. Some of these results were presented in the International Conference on Polynomial and Tensor Optimization, which was held in the city of Xiangtan,

Jiawang Nie

njw@math.ucsd.edu

1 University of California, San Diego, 9500 Gilman Drive, MC 0112, La Jolla, CA 92093, USA 
Hunan Province, China, December 17-21, 2018. The conference was organized by School of Mathematics and Computing Science, Xiangtan University. We would like to warmly thank the organizers: Yunqing Huang, Huazhong Tang, Yin Yang, Liu Yang, and Guangming Zhou, from Xiangtan University. The administration, faculty, staff and students from School of Mathematics and Computing Science, Xiangtan University, enthusiastically accommodated the conference and strongly supported the research of polynomial and tensor optimization. 